\title{
NUMERICAL SOLUTION OF THE TIME-DOMAIN MAXWELL EQUATIONS USING HIGH-ACCURACY FINITE-DIFFERENCE METHODS*
}

\author{
H. M. JURGENS ${ }^{\dagger}$ AND D. W. ZINGG ${ }^{\dagger}$
}

\begin{abstract}
High-accuracy finite-difference schemes are used to solve the two-dimensional timedomain Maxwell equations for electromagnetic wave propagation and scattering. The high-accuracy schemes consist of a seven-point spatial operator coupled with a six-stage Runge-Kutta time-marching method. Two methods are studied, one of which produces the maximum order of accuracy and one of which is optimized for propagation distances smaller than roughly 300 wavelengths. Boundary conditions are presented which preserve the accuracy of these schemes when modeling interfaces between different materials. Numerical experiments are performed which demonstrate the utility of the high-accuracy schemes in modeling waves incident on dielectric and perfect-conducting scatterers using Cartesian and curvilinear grids. The high-accuracy schemes are shown to be substantially more efficient, in both computing time and memory, than a second-order and a fourth-order method. The optimized scheme can lead to a reduction in error relative to the maximum-order scheme, with no additional expense, especially when the number of wavelengths of travel is large.
\end{abstract}

Key words. computational electromagnetics, finite-difference schemes, wave propagation, phase error, Maxwell's equations

AMS subject classifications. 78M20, 65M06

PII. S1064827598334666

1. Introduction. Numerical simulation of the propagation and scattering of electromagnetic waves has a wide range of applications in science and engineering, including antennas, microwave circuits, high-speed digital interconnects, all-optical devices, and many more [23]. The appropriate numerical algorithm for such simulations is dependent on the nature of the system being modeled. For geometries of low electrical size, i.e., spanning at most a few wavelengths, the method of moments is an efficient technique. However, scaling arguments presented by Petropoulos [18] show that this approach quickly becomes impractical as the electrical size increases. Geometries of moderate electrical size can be handled effectively using several available numerical methods for solving the Maxwell equations in the time domain. For such simulations, the geometric flexibility of the finite-element method can compensate for its relative inefficiency for hyperbolic equations. More efficient methods, which are effective for propagation distances on the order of 10 or 20 wavelengths, include the finite-difference methods of Yee [28] and Shang [20] and the finite-volume method of Mohammadian, Shankar, and Hall [16]. However, for propagation distances greater than 20 wavelengths, these methods, which are second-order accurate, typically require excessive grid densities with correspondingly large computational requirements.

The limitations of second-order methods in simulating wave phenomena have led to the development and application of higher-order and optimized finite-difference methods in several fields, including acoustics [13, 24] and seismology [10], as well as electromagnetics [12, 21, 25, 27, 29, 31, 34]. Higher-order methods offer increased accuracy for a given node density at the expense of increased cost per node. Optimized

\footnotetext{
* Received by the editors February 27, 1998; accepted for publication (in revised form) June 14, 2000; published electronically December 20, 2000.

http://www.siam.org/journals/sisc/22-5/33466.html

$\dagger$ Institute for Aerospace Studies, University of Toronto, 4925 Dufferin Street, Downsview, ON, Canada M3H 5T6 (hjurgens@celestica.com,dwz@oddjob.utias.utoronto.ca).
} 
schemes sacrifice the order of accuracy in return for low error over an increased range of wavenumbers. Fourier analysis provides a simple means of analyzing the phase and amplitude errors of finite-difference methods [14, 26, 31, 34]. Several high-order and optimized schemes were compared by Zingg [30] using this approach. Zingg showed that one of the optimized compact schemes developed by Haras and Ta'asan [6] is capable of accurate simulations involving 200 wavelengths of travel with less than 4 grid points per wavelength. This scheme requires the solution of a pentadiagonal system of equations in each coordinate direction. The optimized noncompact schemes of Lockard, Brentner, and Atkins [15] and Zingg, Lomax, and Jurgens [34, 35] require less than 10 grid points per wavelength for 200 wavelengths of travel with a much lower cost per node. Numerical experiments are required to determine the relative efficiencies of these methods in a practical context.

In order to avoid the use of a very small time step, the accuracy of the timemarching method should be comparable to that of the spatial operator. For simulating wave phenomena, Runge-Kutta and Adams-Bashforth methods are natural candidates, with the former generally preferred due to their low memory requirements. For linear ordinary differential equations with constant coefficients, Runge-Kutta methods of up to fourth order require only two memory locations per dependent variable [31]. Five- and six-stage methods with the same memory requirement have been proposed by Haras and Ta'asan [6] and Zingg, Lomax, and Jurgens [34, 35].

The need for stable and accurate numerical boundary schemes presents a major obstacle in the application of high-order finite-difference methods. Since long propagation distances are generally associated with multiple interactions with media interfaces, the numerical boundary schemes can have a significant impact on the overall accuracy of a simulation, and hence on the relative accuracy of various methods. Progress in the development of numerical boundary schemes was reported in $[4$, 17, 33]. Another important consideration in the application of high-order methods is the choice of gridding strategy. Uniform Cartesian grids were used, for example, by Taflove and co-workers [23], while body-fitted curvilinear grids were favored by Shankar, Mohammadian, and Hall [22] and others. The Cartesian approach produces two significant advantages in the interior of the domain. Virtually all numerical methods are most accurate on a uniform grid. In addition, the need to deal with the metrics of a curvilinear coordinate transformation leads to a substantial penalty in terms of both speed and memory. On the other hand, it is extremely difficult to develop stable and accurate boundary treatments for higher-order methods on Cartesian grids. Furthermore, some geometries, such as a curved surface with a thin coating, are not well suited to Cartesian grids. Clearly, the choice of a gridding strategy is problem dependent.

A further issue in the development of numerical methods for the time-domain Maxwell equations is the need to truncate the domain, which inevitably leads to spurious reflections. Boundary conditions based on locally one-dimensional characteristic splitting generally produce excessive reflection. Thus, development of nonreflecting boundary conditions for hyperbolic problems has been an active area of research for many years. Important contributions have been made by Engquist and Majda [5], Bayliss and Turkel [2], Berenger [3], and Petropoulos, Zhao, and Cangellaris [19].

This paper presents the implementation and validation of the high-order and optimized finite-difference methods presented in $[34,35]$ for the solution of the timedomain Maxwell equations. The objective is to demonstrate the efficiency of these methods on nontrivial sample problems. The methods combine a noncompact spatial 
operator with a seven-point stencil and a low-storage six-stage time-marching method. Two methods are studied, one of which produces the maximum order of accuracy and one of which is optimized for propagation distances less than roughly 300 wavelengths. In the next section, the Maxwell equations are presented in generalized curvilinear coordinates. The two finite-difference methods, including both spatial and temporal operators, are then presented. This is followed by a description of the treatment of interfaces and boundaries, including dielectric interfaces, perfect conductors, and domain boundaries. The two methods are applied to a number of test problems involving the propagation and scattering of electromagnetic waves. In order to demonstrate the efficiency of the present methods, the computational requirements are compared with two other methods, one fourth order in space, the other second order.

2. Governing equations. In two dimensions, Maxwell's equations decouple into two sets, the transverse magnetic (TM) and the transverse electric (TE) equations. Without any loss of physics, we consider only the TM set. We restrict our attention to linear, isotropic, perfect dielectric materials with constant properties, no charge density, and no current sources. Under these conditions, the TM equations can be written in the following form:

$$
\frac{\partial \mathbf{Q}}{\partial t}+\mathbf{A} \frac{\partial \mathbf{Q}}{\partial x}+\mathbf{B} \frac{\partial \mathbf{Q}}{\partial y}=\mathbf{0} .
$$

The vector of unknowns is given by

$$
\mathbf{Q}=\left[\begin{array}{c}
D_{z} \\
B_{x} \\
B_{y}
\end{array}\right],
$$

where $D_{z}$ is the $z$ component of the electric flux density, $B_{x}$ and $B_{y}$ are the $x$ and $y$ components of the magnetic flux density, and the two matrices are

$$
\mathbf{A}=\left[\begin{array}{ccc}
0 & 0 & -\frac{1}{\mu} \\
0 & 0 & 0 \\
-\frac{1}{\varepsilon} & 0 & 0
\end{array}\right], \quad \mathbf{B}=\left[\begin{array}{ccc}
0 & \frac{1}{\mu} & 0 \\
\frac{1}{\varepsilon} & 0 & 0 \\
0 & 0 & 0
\end{array}\right]
$$

$\mu$ is the magnetic permeability and $\varepsilon$ is the electric permittivity. The constitutive relations are $\mathbf{D}=\varepsilon \mathbf{E}$ and $\mathbf{B}=\mu \mathbf{H}$, where $\mathbf{E}$ is the electric field intensity and $\mathbf{H}$ is the magnetic field intensity. In our tests, we assume a nondimensional form of the equations where, in free space, $\varepsilon=\mu=1$. Note that the divergence relations are not explicitly enforced. However, numerous tests with the numerical methods studied in this paper show that the divergence of the magnetic field remains of the order of the truncation error for all time.

When numerically modeling electromagnetic fields, it is often the case that the geometry of the problem lends itself to a more generalized coordinate system than Cartesian. A method that allows the independent variables of the partial differential equations to be transformed into a uniformly spaced computational domain was given in [1]. The two-dimensional TM form of Maxwell's curl equations can be expressed in a generalized coordinate system using the following transformation:

$$
\xi=\xi(x, y), \quad \eta=\eta(x, y) .
$$


This allows the Cartesian coordinates to be transformed into curvilinear coordinates in such a way that a curvilinear grid will map to a uniform and square computational space with $\Delta \xi=\Delta \eta=1$. The mapping is usually defined by assigning integer curvilinear coordinate values to each node in the grid and calculating the metrics of the transformation numerically. The transformed equations can be written in the following form:

$$
\frac{\partial \mathbf{Q}}{\partial t}+\hat{\mathbf{A}} \frac{\partial \mathbf{Q}}{\partial \xi}+\hat{\mathbf{B}} \frac{\partial \mathbf{Q}}{\partial \eta}=\mathbf{0}
$$

where the two matrices are

$$
(2.6) \hat{\mathbf{A}}=\left[\begin{array}{ccc}
0 & \frac{1}{\mu} \frac{\partial \xi}{\partial y} & -\frac{1}{\mu} \frac{\partial \xi}{\partial x} \\
\frac{1}{\varepsilon} \frac{\partial \xi}{\partial y} & 0 & 0 \\
-\frac{1}{\varepsilon} \frac{\partial \xi}{\partial x} & 0 & 0
\end{array}\right], \quad \hat{\mathbf{B}}=\left[\begin{array}{ccc}
0 & \frac{1}{\mu} \frac{\partial \eta}{\partial y} & -\frac{1}{\mu} \frac{\partial \eta}{\partial x} \\
\frac{1}{\varepsilon} \frac{\partial \eta}{\partial y} & 0 & 0 \\
-\frac{1}{\varepsilon} \frac{\partial \eta}{\partial x} & 0 & 0
\end{array}\right]
$$

Extension to spatially variable material properties requires the use of a strongconservation-law form of the equations, as discussed in [11].

3. Numerical method. Our aim is to solve for the total electric and magnetic field components by approximating Maxwell's equations using finite-difference methods. The finite-difference schemes used consist of a seven-point spatial operator in conjunction with an explicit six-stage time-marching method. These high-accuracy methods were originally presented by Zingg, Lomax, and Jurgens [35], and an extensive description of the methods, including an error and stability analysis, can be found in [34].

The spatial operator is made up of two parts, an antisymmetric or centraldifference operator,

$$
\left(\delta_{x}^{a} u\right)_{j}=\frac{1}{\Delta x}\left[a_{1}\left(u_{j+1}-u_{j-1}\right)+a_{2}\left(u_{j+2}-u_{j-2}\right)+a_{3}\left(u_{j+3}-u_{j-3}\right)\right],
$$

and a symmetric operator,

$$
\left(\delta_{x}^{s} u\right)_{j}=\frac{1}{\Delta x}\left[d_{0} u_{j}+d_{1}\left(u_{j+1}+u_{j-1}\right)+d_{2}\left(u_{j+2}+u_{j-2}\right)+d_{3}\left(u_{j+3}+u_{j-3}\right)\right] .
$$

The operator described by (3.1) has a maximum formal order which varies as $\Delta x^{6}$ when $a_{1}=\frac{3}{4}, a_{2}=-\frac{3}{20}$, and $a_{3}=\frac{1}{60}$. The operator described by (3.2) has a maximum formal order which varies as $\Delta x^{5}$ when $d_{1}=-\frac{3}{4} d_{0}, d_{2}=\frac{3}{10} d_{0}$, and $d_{3}=-\frac{1}{20} d_{0}$. The symmetric operator is used to add a small amount of numerical dissipation to the scheme. We use a value of $d_{0}=\frac{1}{10}$. A characteristic splitting is used when the operator is applied to a hyperbolic system of equations. For example, in order to approximate the term $\mathbf{A} \frac{\partial \mathbf{Q}}{\partial x}$, the scheme is applied as follows:

$$
\mathbf{A}\left(\frac{\partial \mathbf{Q}}{\partial x}\right)_{j}=\mathbf{A}\left(\delta_{x}^{a} \mathbf{Q}\right)_{j}+|\mathbf{A}|\left(\delta_{x}^{s} \mathbf{Q}\right)_{j}
$$

at any interior node $j$. Note that $|\mathbf{A}|=\mathbf{X}|\Lambda| \mathbf{X}^{-1}$, where $\mathbf{X}$ is the matrix of right eigenvectors of $\mathbf{A}$, and $\Lambda$ is the matrix of eigenvalues of $\mathbf{A}$. The method is applied in a similar way in order to approximate the $y$-derivatives of the field values. 
The time-marching method, when applied to the ordinary differential equation $\frac{d u}{d t}=f(u, t)$, can be written as

$$
\begin{aligned}
& u_{n+\alpha_{1}}^{(1)}=u_{n}+h \alpha_{1} f_{n}, \\
& u_{n+\alpha_{2}}^{(2)}=u_{n}+h \alpha_{2} f_{n+\alpha_{1}}^{(1)}, \\
& u_{n+\alpha_{3}}^{(3)}=u_{n}+h \alpha_{3} f_{n+\alpha_{2}}^{(2)}, \\
& u_{n+\alpha_{4}}^{(4)}=u_{n}+h \alpha_{4} f_{n+\alpha_{3}}^{(3)}, \\
& u_{n+\alpha_{5}}^{(5)}=u_{n}+h \alpha_{5} f_{n+\alpha_{4}}^{(4)}, \\
& u_{n+1}=u_{n}+h f_{n+\alpha_{5}}^{(5)},
\end{aligned}
$$

where $h=\Delta t$ is the time step, $t_{n}=n h, u_{n}=u\left(t_{n}\right)$, and $f_{n+\alpha}^{(k)}=f\left(u_{n+\alpha}^{(k)}, t_{n}+\alpha h\right)$. Setting $\alpha_{5}=\frac{1}{2}$ gives a method that is second-order accurate. Additionally setting $\alpha_{4}=\frac{1}{3}, \alpha_{3}=\frac{1}{4}, \alpha_{2}=\frac{1}{5}$, and $\alpha_{1}=\frac{1}{6}$ gives the method with the highest formal order for linear homogeneous ordinary differential equations, which is sixth-order accurate. For inhomogeneous and nonlinear ordinary differential equations, it is second-order accurate. Two memory locations are required per dependent variable. The stability region for this method was shown in [34].

The above operators contain coefficients that can be determined in a number of ways. We have already described the operators in which the coefficients are determined by maximizing the formal order (referred to as the maximum-order (MO) operator). Zingg, Lomax, and Jurgens [34, 35] presented an additional method, in which the coefficients are determined by specifying that the phase and amplitude errors of the operators are minimized for waves which are resolved by the computational grid with at least 10 points per wavelength (referred to as the optimized operator or O10). The coefficients for this optimized scheme can be found in the appendix.

These schemes have a number of useful properties. First, the amplitude error is less than the phase error for all wavenumbers [34]. Hence any wavenumber components that are excessively damped also have excessive phase error. Second, the timemarching method produces significantly smaller errors than the spatial discretization. Hence the phase and amplitude errors are virtually independent of the Courant number (for Courant numbers less than unity). This contrasts with some other schemes that are accurate only within a narrow range of Courant numbers. Similarly, the present schemes do not require that the direction of wave propagation be aligned with the grid. The errors are largest for propagation angles of zero and 90 degrees. Hence the one-dimensional analysis given in [34] gives an upper bound on the error.

4. Interface and boundary treatment. In order to simulate the scattering of electromagnetic waves off of materials which have differing dielectric properties, each region is modeled as a distinct numerical domain. These domains are then coupled using the appropriate physical boundary conditions. This procedure allows the grids for each region to be generated independently, which is useful since the resolution of the grid is dependent on the material properties of the domain. Three separate boundary types are considered, an interface between two dielectric regions, the boundary between a dielectric and a perfect conductor, and the far-field boundary which is located at the outer region of the domain. At the interface between two dielectric media and at the surface of a perfect conductor, a locally one-dimensional characteristic formulation of the governing equations is used [8]. Using this approach, 
characteristic variables can be found that represent incoming and outgoing waves along lines normal to the interface. For example, if we consider a boundary that lies at the right side of the domain on a line of constant $x$, the incoming waves are associated with $\mathbf{A}^{-}$and the outgoing waves with $\mathbf{A}^{+}$, where

$$
\mathbf{A}^{ \pm}=\frac{\mathbf{A} \pm|\mathbf{A}|}{2}
$$

and $|\mathbf{A}|$ is defined after (3.3). Similar expressions can be found for boundaries that lie on lines of constant $y$ and for boundaries in curvilinear coordinates. For the curvilinear case, the incoming and outgoing waves will be associated with $\hat{\mathbf{A}}^{ \pm}$and $\hat{\mathbf{B}}^{ \pm}$. When calculating spatial derivatives near boundaries and interfaces, flux-vector splitting is used. Regular fifth-order one-sided and upwind-biased schemes are used for the outgoing waves, while the following numerical boundary scheme (NBS) is used for the incoming waves:

$$
\begin{aligned}
& \left(\delta_{x} u\right)_{1}=\frac{1}{60 \Delta x}\left[-3 u_{0}-119 u_{1}+255 u_{2}-240 u_{3}+155 u_{4}-57 u_{5}+9 u_{6}\right] \\
& \left(\delta_{x} u\right)_{2}=\frac{1}{60 \Delta x}\left[9 u_{0}-66 u_{1}+70 u_{2}-60 u_{3}+75 u_{4}-34 u_{5}+6 u_{6}\right] .
\end{aligned}
$$

The above NBS is a modified version of the one presented by Zingg, Lomax, and Jurgens $[34,35]$, which was found to be unstable on some curvilinear grids.

4.1. Dielectric interfaces. On the interface between two dielectrics the following conditions must hold:

$$
\begin{aligned}
& E_{t}^{(1)}=E_{t}^{(2)}, \\
& H_{t}^{(1)}=H_{t}^{(2)}, \\
& D_{n}^{(1)}=D_{n}^{(2)}, \\
& B_{n}^{(1)}=B_{n}^{(2)} .
\end{aligned}
$$

The superscripts refer to the field values in materials (1) and (2). Note that these relations assume that there is no surface current flowing along the interface and that there is no surface charge density present at the interface. In the TM case, the electric intensity and electric flux density vectors point out of the $x-y$ plane, and thus (4.5) holds automatically and (4.3) simplifies to

$$
E_{z}^{(1)}=E_{z}^{(2)}
$$

Consider a body-fitted grid which ensures that the interface between different materials will lie on a $\xi=$ constant curve. The grid is constrained to be orthogonal near the body, thus $\eta=$ constant curves are normal to the interface. In this example, we assume that the positive $\xi$ direction points from region (1) to region (2). Figure 1 shows an example of such a body-fitted coordinate system. We can generalize this example to different cases in curvilinear coordinates and to Cartesian coordinates quite easily. There are six unknown values which need to be stored on the interface: $D_{z}^{(1)}, D_{z}^{(2)}, B_{x}^{(1)}, B_{x}^{(2)}, B_{y}^{(1)}$, and $B_{y}^{(2)}$. The fields are extrapolated to the interface using a sixth-order method (see the appendix). The characteristic variables are then calculated from

$$
w_{+}^{(1)}=Z^{(1)} D_{z}^{(1)}+B_{t}^{(1)}
$$




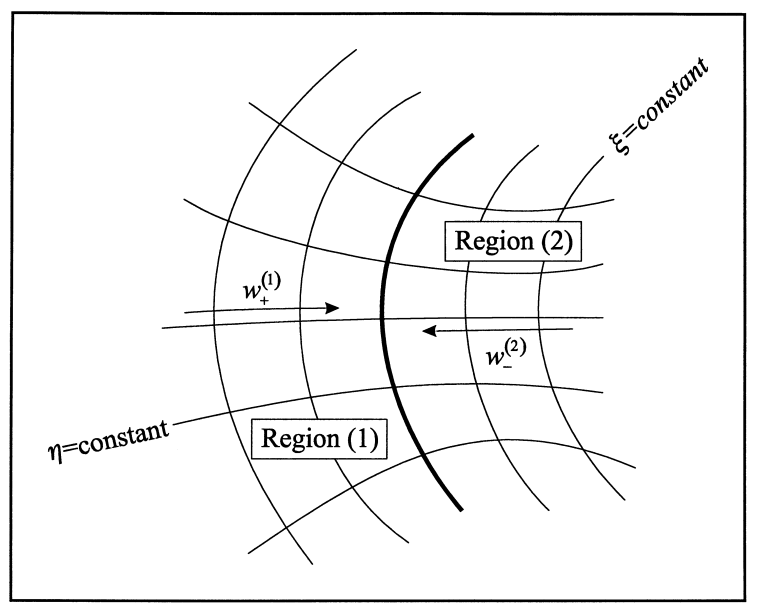

FIG. 1. Example of a body-fitted grid showing the characteristic variables associated with the outgoing waves.

and

$$
w_{-}^{(2)}=Z^{(2)} D_{z}^{(2)}-B_{t}^{(2)},
$$

where $Z=(\mu / \varepsilon)^{1 / 2}$ is the intrinsic impedance of the medium. Note that

$$
B_{t}=\frac{\frac{\partial \xi}{\partial y} B_{x}-\frac{\partial \xi}{\partial x} B_{y}}{\left[\left(\frac{\partial \xi}{\partial x}\right)^{2}+\left(\frac{\partial \xi}{\partial y}\right)^{2}\right]^{1 / 2}}
$$

is the component of $\mathbf{B}$ tangent to a $\xi=$ constant curve, and

$$
B_{n}=\frac{\frac{\partial \xi}{\partial x} B_{x}+\frac{\partial \xi}{\partial y} B_{y}}{\left[\left(\frac{\partial \xi}{\partial x}\right)^{2}+\left(\frac{\partial \xi}{\partial y}\right)^{2}\right]^{1 / 2}}
$$

is the component of $\mathbf{B}$ normal to a $\xi=$ constant curve. This gives two equations for the unknown values $D_{z}^{(1)}, D_{z}^{(2)}, B_{t}^{(1)}$, and $B_{t}^{(2)}$. Note that the quantities extrapolated depend on the orientation of the local $(\xi, \eta)$ coordinate system at the interface. Combining these with the jump conditions, we obtain

$$
\begin{gathered}
D_{z}^{(1)}=\frac{w_{+}^{(1)}+\frac{\mu^{(1)}}{\mu^{(2)}} w_{-}^{(2)}}{Z^{(1)}\left(1+\frac{Z^{(1)}}{Z^{(2)}}\right)}, \\
D_{z}^{(2)}=\frac{\varepsilon^{(2)}}{\varepsilon^{(1)}} D_{z}^{(1)},
\end{gathered}
$$




$$
\begin{gathered}
B_{t}^{(1)}=\frac{Z^{(1)} w_{+}^{(1)}-\mu^{(1)} c^{(2)} w_{-}^{(2)}}{Z^{(1)}+Z^{(2)}}, \\
B_{t}^{(2)}=\frac{\mu^{(2)}}{\mu^{(1)}} B_{t}^{(1)} .
\end{gathered}
$$

The variable $c=(\varepsilon \mu)^{-1 / 2}$ is the propagation speed. The normal component of the magnetic flux density is single valued on the interface, as shown by (4.6), and is determined by averaging the two values obtained by extrapolation. The values of $B_{x}$ and $B_{y}$ are determined from $B_{n}$ and $B_{t}$ using (4.10) and (4.11).

The above interface treatment was closely examined in [32]. The error introduced at interfaces is of the same order of magnitude as that introduced in the interior using the high-accuracy schemes. Both the interior discretization and the interface treatment, including the NBS (4.2), contribute to the overall error, with the relative contribution depending on the distance between interfaces.

4.2. Perfect conductors. At the interface between a dielectric material and a perfect conductor, the physical boundary conditions given in the above section must be modified. If, for example, region (2) is a perfect conductor - that is, having an infinite conductivity - then all the field values inside the region must be zero. Using this fact, and the four relations given in the above section, one obtains the following boundary conditions at the surface of a perfect conductor:

$$
\begin{aligned}
E_{t} & =0, \\
H_{t} & =J_{s}, \\
D_{n} & =0, \\
B_{n} & =0 .
\end{aligned}
$$

In general, there will always be a finite current density, $J_{s}$, on the surface of a perfect conductor, but we have assumed a zero surface charge density. For the TM case, the third condition is automatically satisfied since the electric flux density points out of the plane, and the first condition sets its magnitude to zero, i.e., $D_{z}=0$. The tangential component of the magnetic flux density, and thus the surface current, is found by extrapolating the fields to the surface and then calculating the outgoing characteristic variable. For example, if the surface of the perfect conductor lies along a grid line of constant $\xi$, with $\xi$ increasing as the boundary is approached, then $w_{+}$ is determined from (4.8). Since $D_{z}=0,(4.8)$ gives

$$
B_{t}=w_{+}
$$

where $B_{t}$ is defined by (4.10). The Cartesian components of the magnetic flux density are determined using this value and the fact that $B_{n}=0$.

4.3. Far-field boundaries. To model the problem of waves moving out into free space, the numerical domain must be artificially truncated. There is a considerable body of literature on the subject of far-field boundary conditions which tries to minimize the spurious reflection introduced when a disturbance propagates out of the numerical domain $[2,3,5,19,23,24]$. There are many papers, such as [9], that discuss the merits of different types of far-field conditions.

We implement a modified version of the radiating boundary condition introduced by Bayliss and Turkel [2], which was found to be the best method of those tested by 
Hixon, Shih, and Mankbadi [9]. This method truncates the domain by using a farfield radiation condition which only allows disturbances to propagate in the outward direction. The condition is applied by enforcing the following partial differential equation on the boundary region of the domain:

$$
\left(\frac{1}{c} \frac{\partial}{\partial t}+\frac{\partial}{\partial r}+\frac{a}{2 r}\right)\left[\begin{array}{c}
D_{z} \\
B_{x} \\
B_{y}
\end{array}\right]=\mathbf{0}
$$

When $a=1$ the operator given in [2] is recovered, which is $\mathrm{O}\left(r^{-5 / 2}\right)$. Using the operator with this value of $a$ results in the numerical schemes becoming unstable for some curvilinear grids. We use a value of $a=3$, which results in a stable and accurate method. The numerical domain is split into two regions, one of which contains the total electromagnetic field and one of which contains only the scattered field (see, for example, [23]). We store the scattered field on ghost-points which lie outside the domain, and the total field at all other nodes. To approximate the spatial derivatives in Maxwell's equations where the computational stencil will overlap the ghost-point region, the incident field is added to the scattered field at the necessary nodes in this region. When calculating the spatial derivatives for the radiation condition, the incident field is subtracted from the total field at the necessary nodes in the interior domain.

\section{Results and discussion.}

5.1. Dielectric square. We first simulate the scattering of a pulsed plane wave off of a dielectric square. Figure 2 shows an example grid as well as the geometry of the problem. The domain is square, with $x$ and $y$ ranging from 0 to 1 , and the dielectric is located in the center of this region, at $0.4 \leq x \leq 0.6,0.4 \leq y \leq 0.6$. The permittivity of the dielectric is four times that of the free space region. The wavespeed in free space is normalized to one, resulting in a wavespeed of one-half in the dielectric. The domain is discretized using a Cartesian grid, in which $\Delta x=\Delta y$, and the grid density in the interior of the dielectric is twice that of free space. Results are obtained for a Gaussian pulse incident upon the dielectric square at an angle of $45^{\circ}$. The incident electric field is given by

$$
E_{z}(x, y, t)=\exp \left[-\frac{1}{2 \sigma^{2}}\left(x \cos \frac{\pi}{4}+y \sin \frac{\pi}{4}+\frac{1}{2}-t\right)^{2}\right]
$$

with $\sigma=0.03$. For all test cases, the time step is chosen in order to give a Courant number of unity in free space. This results in a value of $\Delta t$ equal to the $\Delta x$ value of the free space domain. As a result of both the wave speed and the grid spacing inside the dielectric being one half the values found outside the dielectric, the Courant number will also have a value of unity inside the dielectric. This ensures that the spatial resolution inside the dielectric is matched with that found in free space, since the width of the pulse will narrow as it slows down. Because of this, the errors will not be as localized to the interior of the dielectric as they were in the tests performed in section 5.1 of [11], where the grid resolution was kept constant over the entire domain. The better grid resolution inside the dielectric also allows for an accurate simulation of a narrower pulse. Note, however, that the accuracy of the high-accuracy finite-difference schemes is equally good at Courant numbers less than unity. Spurious reflections are not an issue for this case because the incident field is imposed along 


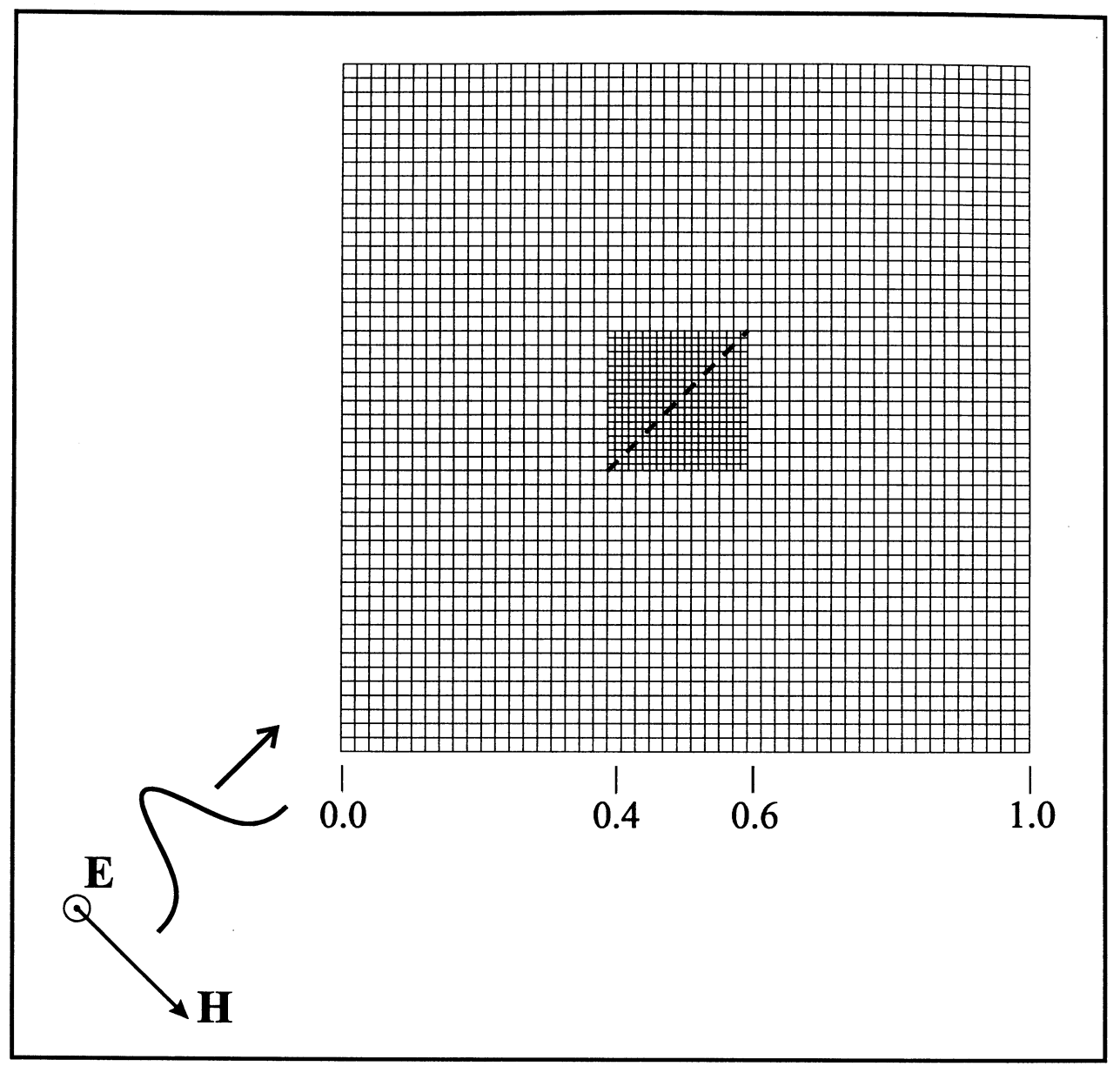

FIG. 2. Example grid showing pulsed plane wave with Gaussian cross-section incident at $45^{\circ}$ on a dielectric square.

the entire outer boundary, and the simulation is not run long enough for the reflected pulse to reach the outer boundary.

For this simulation the interfaces between the different materials lie along lines in the Cartesian grid. The jump conditions discussed in section 4.1 can be used to obtain the field values along grid lines that cross the interfaces. In order to obtain the field unknowns along the interface at nodes inside the dielectric, which do not have a corresponding node outside the dielectric, interpolation and extrapolation of values from neighboring interface nodes are used. The interpolation and extrapolation schemes can be found in the appendix. Note that the extrapolation scheme and the noncentered interpolation schemes are used for the nodes near the corners of the dielectric. The corners must also be treated as special cases. It is only necessary to store the field values at the corner nodes for the free space part of the domain since the values in the interior of the dielectric are never used during the simulation. These values are obtained by taking the average value of the results of interpolating the field values along the $x$ and $y$ grid lines which intersect the corner node. Again, the relevant inerpolation schemes can be found in the appendix. Note that the presence of the 


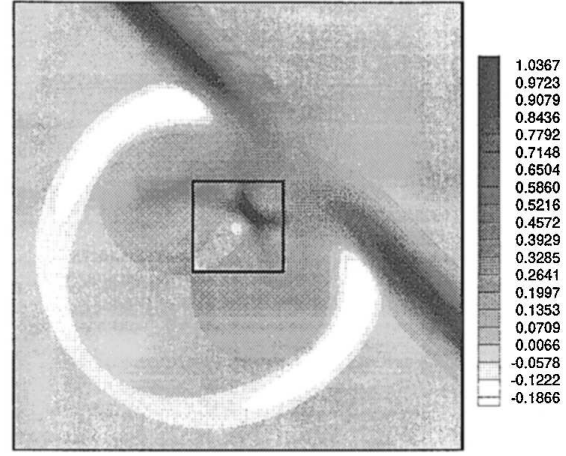

a) reference solution

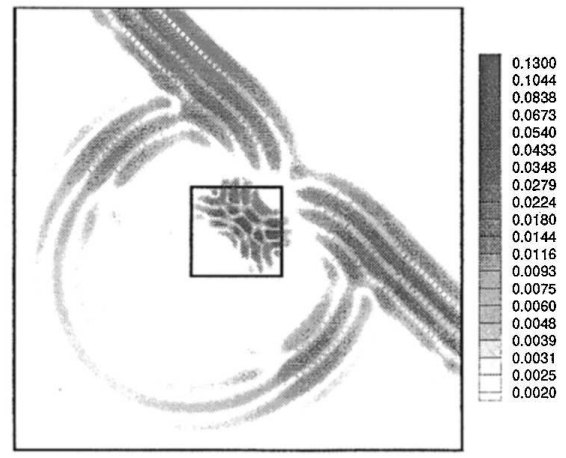

c) $\mathrm{C} 4$ error using 11,200 nodes

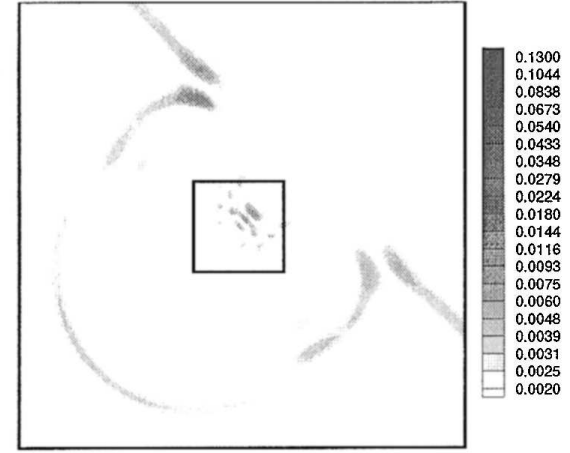

b) MO error using 11,200 nodes

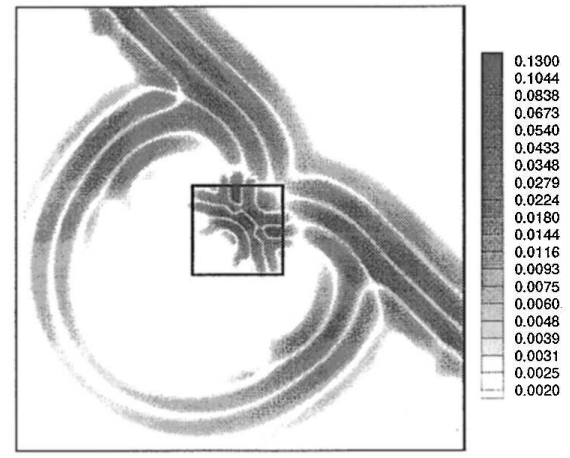

d) C2 error using 44,800 nodes

FIG. 3. Contour plots of electric field intensity and errors in electric field intensity. (a) Reference solution, (b) MO error using 11,200 nodes, (c) C4 error using 11,200 nodes, and (d) C2 error using 44,800 nodes.

singularity in the neighborhood of the corners introduces a low-order error locally. The finite-difference scheme can be modified locally to account for the singularity, but our results will show that the high-accuracy methods produce significant error reduction without implementing such a strategy.

In addition to the MO and $\mathrm{O} 10$ schemes described here, results are presented for second-order centered differences in space combined with fourth-order Runge-Kutta time marching $(\mathrm{C} 2)$ and fourth-order centered differences in space combined with fourth-order Runge-Kutta time marching (C4).

Contour plots of the absolute value of the error in the electric field intensity at $t=1.4$ are shown for three different cases in Figure 3. Figure 3(a) shows the electric field intensity for a reference solution calculated using the MO scheme on a grid with 179,200 nodes. The error obtained using the MO scheme on a grid with 11,200 nodes is shown in Figure 3(b). Note that the contour levels grow exponentially, and the error is extremely small. Plots of the errors obtained using method $\mathrm{C} 4$ on the grid with 


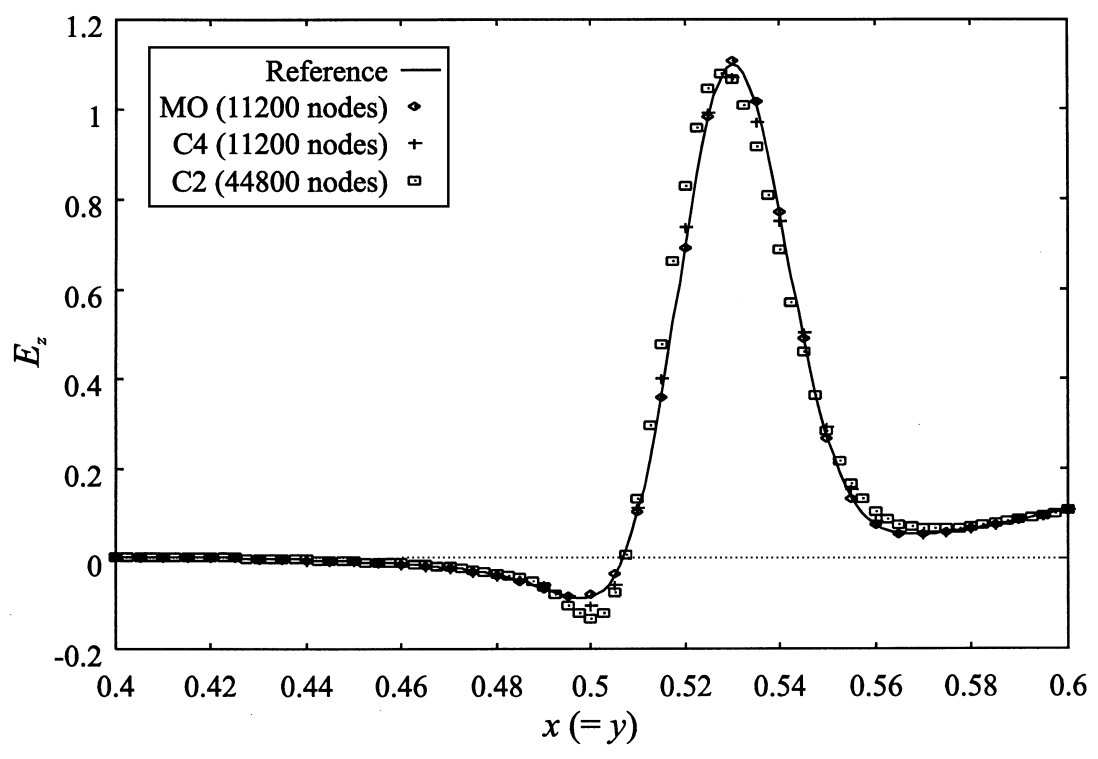

FIG. 4. Electric field intensity across diagonal of dielectric square.

11,200 nodes and method C2 on a grid with 44,800 nodes are shown in Figures 3(c) and (d), respectively. The errors obtained using method $\mathrm{C} 4$ are much larger than those obtained using the MO scheme, and even greater errors are evident in the $\mathrm{C} 2$ results, which are computed on a grid with four times as many nodes.

The electric field intensity along the diagonal of the dielectric square is shown in Figure 4 for the reference solution, the MO scheme on a grid with 11,200 nodes, the C4 scheme on a grid with 11,200 nodes, and the C2 scheme on a grid with 44,800 nodes. The results for the MO scheme lie very close to the reference solution. The C4 results are also fairly accurate, but there are some discernible deviations from the reference solution near the maximum and minimum field values. The solution obtained using $\mathrm{C} 2$ deviates considerably from the reference values.

Figure 5 compares the $\mathrm{L}_{2}$ norm of the error in the electric field intensity, normalized by the number of nodes in the numerical domain, versus the number of nodes, for the $\mathrm{C} 2, \mathrm{C} 4, \mathrm{MO}$, and $\mathrm{O} 10$ schemes. The errors are determined by considering the results obtained from a simulation on the highly resolved grid as an accurate reference solution. Since the number of nodes is inversely proportional to the mesh spacing squared, the $\mathrm{C} 2$ and $\mathrm{C} 4$ schemes display roughly second- and fourth-order convergence, respectively. This indicates that the effect of the corner singularity is not large. The MO and O10 schemes, which are formally second order as a result of the time-marching method, produce fourth-order accuracy over the range shown. However, our emphasis here is not on the asymptotic error behavior but on the grid density required to achieve specified error levels. The high-accuracy schemes show a significant advantage in this regard.

Figure 6 plots the error as a function of the computer time needed to obtain the solution. All schemes are implemented in a consistent manner. Hence computing time is an objective measure of cost, and similar relative performance is expected across computing platforms. It is clear that the high-accuracy schemes (MO and O10) are more efficient than the $\mathrm{C} 4$ and $\mathrm{C} 2$ schemes. To obtain the accuracy of the MO and 


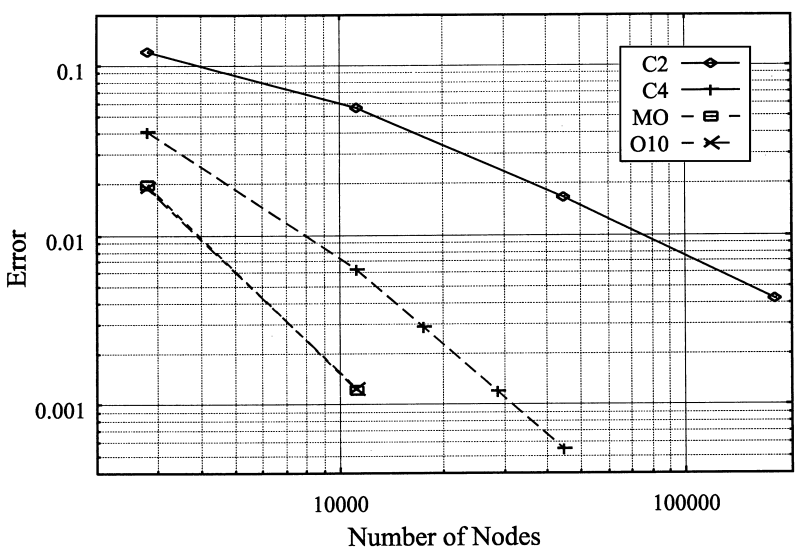

FIG. 5. $\mathrm{L}_{2}$ norm of the error in the electric field intensity for the dielectric square case, as a function of the number of nodes in the grid.

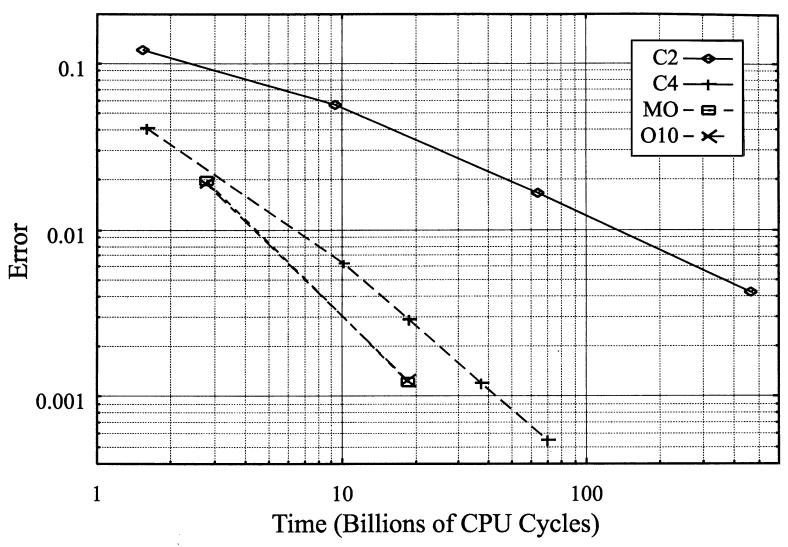

FIG. 6. $\mathrm{L}_{2}$ norm of the error in the electric field intensity for the dielectric square case, as a function of the CPU cycles necessary to complete simulation.

O10 methods on a grid with 11,200 nodes, the C4 scheme needs to be run on a grid with 2.5 times as many grid nodes, requiring twice as much CPU time. The results obtained using $\mathrm{C} 2$ on a grid with 16 times as many nodes gives an error which is about 3.5 times as large as that obtained by the MO scheme, taking about 25 times longer to run. Even though the high-accuracy schemes take longer to run per grid node per time step, this is easily offset by the fact that smaller errors can be obtained by using a grid with far fewer nodes, and, as a result, a larger time step. The savings in memory is a direct result of using a grid with fewer nodes. The savings in both time and memory will be even more evident in three dimensions.

For the above cases the optimized method (O10) gives the same results as the MO scheme. This is primarily due to the nature of the Gaussian pulse, which has significant low wavenumber content. The majority of the Fourier components of the pulse have wavenumbers that give a value of $\kappa \Delta x<0.35$ on the grid with 11,200 nodes. These are resolved more accurately by the MO scheme. 


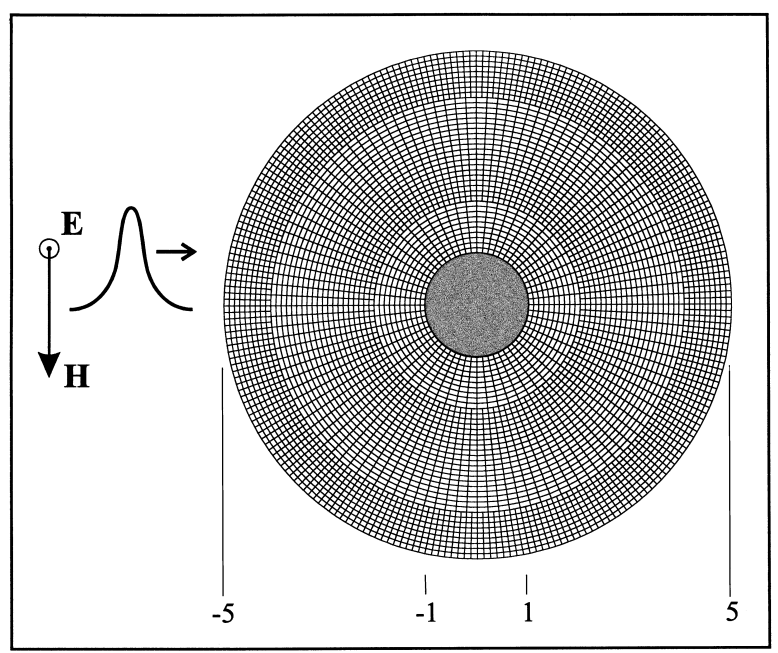

FIG. 7. Example grid showing pulsed plane wave with Gaussian cross-section incident on a perfectly conducting cylinder.

5.2. Perfectly conducting cylinder. We now consider an example using a curvilinear grid which consists of a waveform incident on a perfectly conducting cylinder. Figure 7 shows the geometry of the problem. The cylinder has a radius of unity, and the outer limit of the numerical domain is at $r=5$. The speed at which a wave travels in free space is again normalized to unity. The waveform enters the domain from the left. The simulations described in section 5.2 of [12] are improved by modifying the grids so they are made up of three distinct zones. These grids, an example of which is shown in Figure 7, limit the widening of individual cells as one moves away from the scatterer, which allows the modeling of narrower pulses. This is a result of doubling the resolution of the grid, in the $\theta$ direction, whenever the value of $r$ is doubled. This ensures that the waves are evenly resolved over the entire computational domain. The zones in the domain are allowed to overlap slightly in order to avoid the use of boundary operators where they interface. Interpolation must be used in order to obtain the field values at nodes that do not have corresponding nodes in the zone being overlapped. The interpolation schemes are described in the appendix. The grids are generated in order to obtain cells that have an aspect ratio close to 1 near the perfect conductor. When the aspect ratio deviates too much from this value, it is difficult to find boundary schemes that are stable and accurate for the high-accuracy methods. For all the tests, the time step is chosen so that the Courant number falls in the range 0.5 to 1.0. The grid metrics are calculated numerically using the same antisymmetric operator used in the calculation of the spatial derivatives of the electromagnetic fields. It should be emphasized that such a grid, i.e., one that produces a small range of Courant numbers, is not required in order to demonstrate the efficiency of the high-accuracy schemes, as shown in [12]. We have generated the grid in this manner simply to show that it can easily be done and to avoid a simulation in which the numerical errors are introduced primarily in one region of the grid.

5.2.1. Gaussian incident pulse. Consider a pulsed plane wave with a Gaussian cross-section incident on the cylinder. The incident electric field intensity is given by 


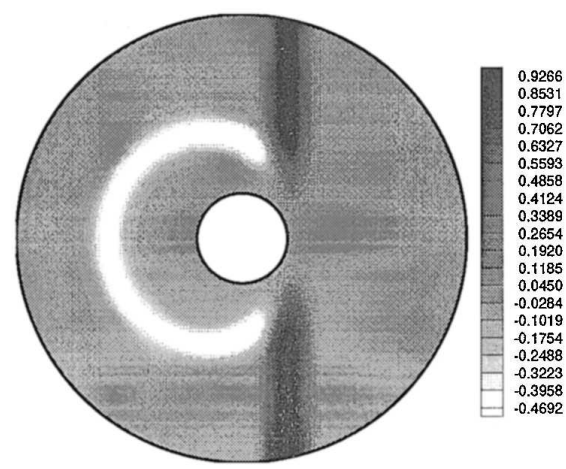

a) reference solution

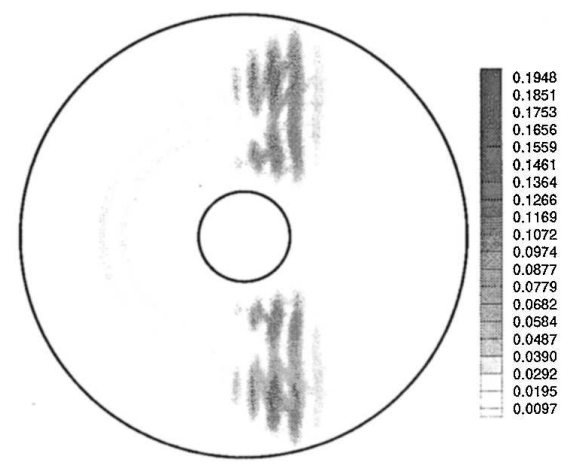

c) C4 error using 5,400 nodes

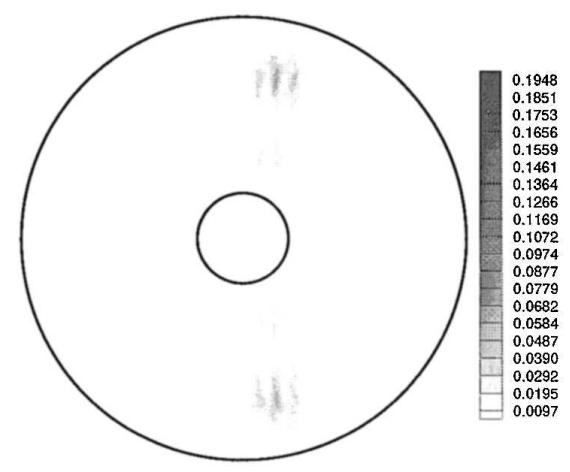

b) MO error using 5,400 nodes

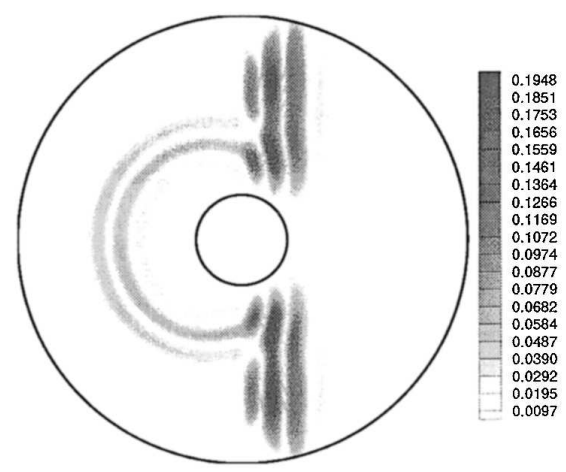

d) C2 error using 21,600 nodes

FIG. 8. Contour plots of electric field intensity and errors in electric field intensity. (a) Reference solution, (b) MO error using 5,400 nodes, (c) C4 error using 5,400 nodes, and (d) C2 error using 21,600 nodes.

$$
E_{z}(x, y, t)=\exp \left[-\frac{1}{2 \sigma^{2}}\left(x+\frac{15}{2}-t\right)^{2}\right]
$$

with $\sigma=0.3$, and the simulation is run until $t=8.5$. Figure 8 (a) shows a contour plot of the electric field intensity for a reference solution calculated using the $\mathrm{MO}$ scheme on a grid with 345,600 nodes. The absolute value of the error obtained using the MO scheme on a grid with 5,400 nodes is shown in Figure 8(b). Even though this grid is fairly coarse, the errors are seen to be very small. Plots of the errors obtained using method $\mathrm{C} 4$ on the grid with 5,400 nodes and method $\mathrm{C} 2$ on a grid with 21,600 nodes are shown in Figures 8(c) and (d), respectively. Both of these solutions show significant deviations from the reference solution.

The electric field intensity along the $x$-axis, behind the perfect conductor, is shown in Figure 9. In this region, the solution is not visible in Figure 8(a) and is very sensitive to numerical errors. The reference solution, the results from the MO 


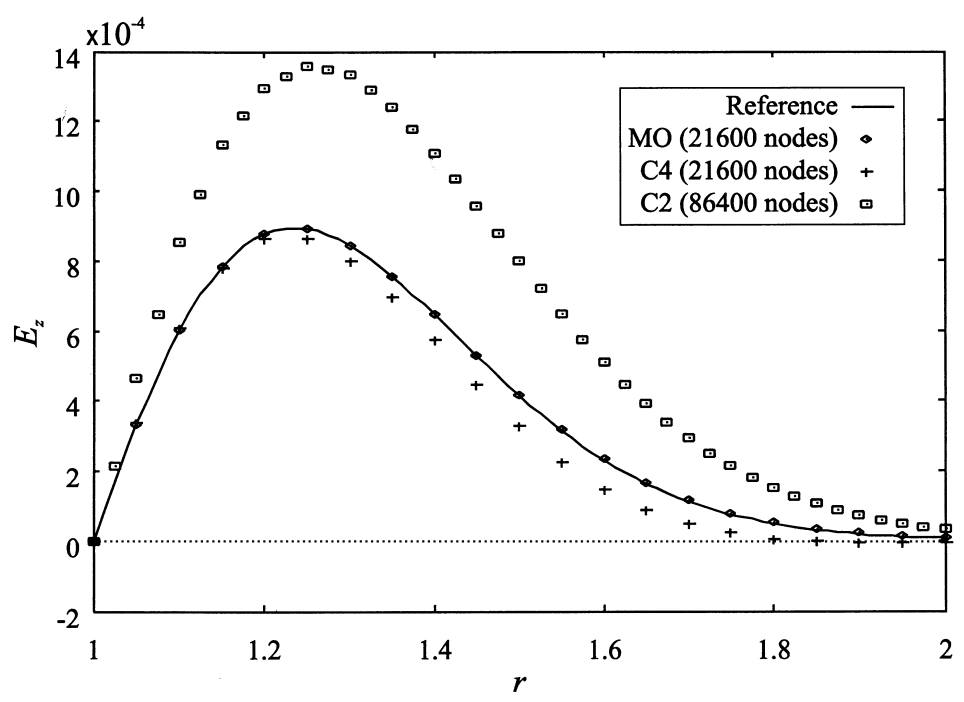

FIG. 9. Electric field intensity along a radial line behind the perfectly conducting cylinder for an incident pulse with a Gaussian cross-section.

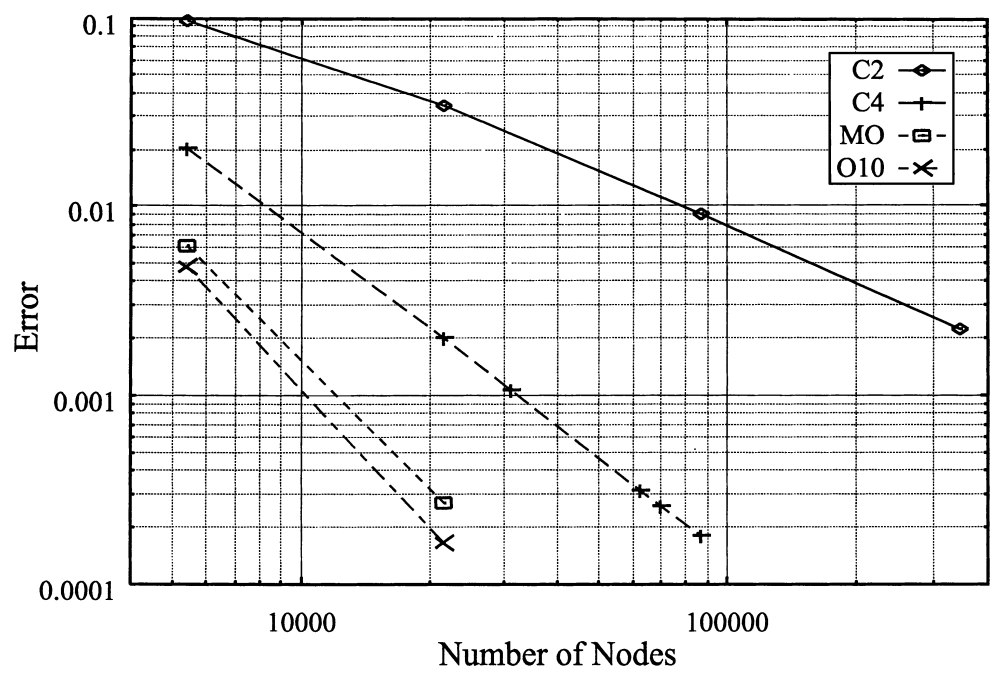

FIG. 10. $\mathrm{L}_{2}$ norm of the error in the electric field intensity as a function of the number of nodes in the grid for a pulsed plane wave incident on the perfectly conducting cylinder.

scheme on a grid having 21,600 nodes, the results from the C4 scheme on a grid with 21,600 nodes, and the results from the C2 scheme on a grid with 86,400 nodes are all shown. The solution obtained using the MO scheme is very accurate. Using the same grid, the C4 method produces a solution that deviates significantly from the reference solution. The solution obtained by using $\mathrm{C} 2$ on a grid with four times as many nodes is not adequate for engineering purposes.

Figure 10 shows the normalized $\mathrm{L}_{2}$ norm of the errors in the electric field intensity as a function of the number of nodes in the grid, and Figure 11 shows the error as a function of the CPU usage. The error value for each test is calculated using a reference 


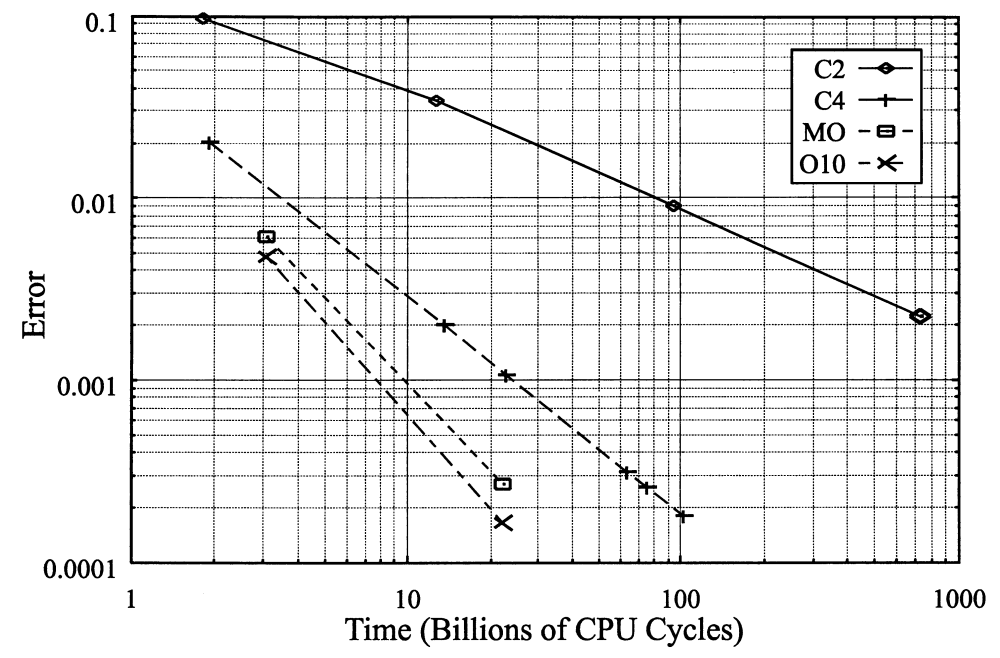

FIG. 11. $\mathrm{L}_{2}$ norm of the error in the electric field intensity as a function of the number of $C P U$ cycles it takes to complete the simulation for a pulsed plane wave incident on the perfectly conducting cylinder.

solution obtained using the MO scheme on a highly resolved grid. The trends shown in these figures are very similar to those obtained for the dielectric square case. To attain the same error level as the MO scheme used on a grid with 21,600 nodes, the C4 scheme must use about 3.25 times as many nodes and takes about 3.4 times as long to complete the simulation. Even when using a grid with 345,600 nodes, the C2 method does not produce a solution that can compare with those computed by the high-accuracy methods on a grid with 21,600 nodes. The O10 scheme is seen to produce slightly better results than the MO scheme when using the same grid. For example, on the grid with 21,600 nodes, the O10 scheme results in an error that is $61 \%$ of that obtained using the MO scheme, with no additional CPU expense.

5.2.2. Cosine incident wave. In this section, simulations of a cosine plane wave incident on the perfectly conducting cylinder are presented. The existence of an analytical solution for a plane harmonic electromagnetic wave incident upon a perfectly conducting circular cylinder is well known and can be found in, for example, [7]. The incident electric field intensity is given by

$$
E_{z}(x, y, t)=\cos \kappa(x-t)
$$

with $\kappa=2 \pi$. A shaded contour plot of the analytical solution for the total electric field intensity is shown in Figure 12.

Figure 13 compares the errors as a function of the number of nodes in the grids. Figure 14 plots these errors as a function of the CPU time needed to complete the simulations. These plots again show the superior efficiency of the high-accuracy methods. The second-order method produces significant errors for all three of the grids. The fourth-order method needs to use the most resolved grid to generate a solution with acceptable accuracy, which can be produced by the high-accuracy methods on a grid with four times fewer nodes. Figure 15 shows the electric field intensity along a radial line directly behind the scatterer for the analytical, fourth-order, and MO solutions. The numerical solutions were obtained on a grid with 21,600 nodes. The solution ob- 


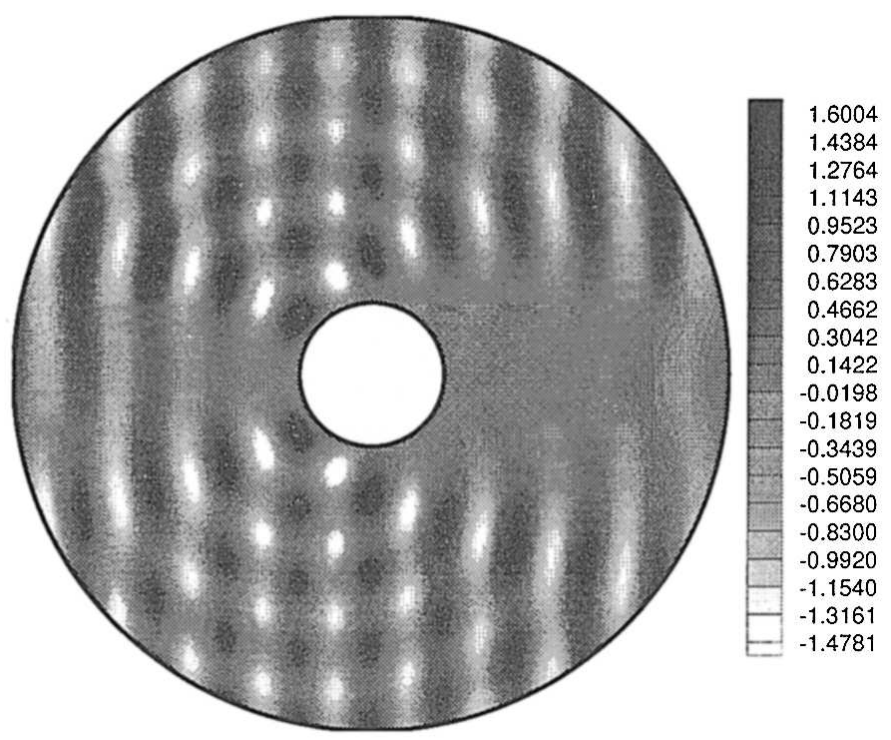

FIG. 12. Electric field intensity for cosine wave, with $\kappa=2 \pi$, incident on a perfectly conducting cylinder.

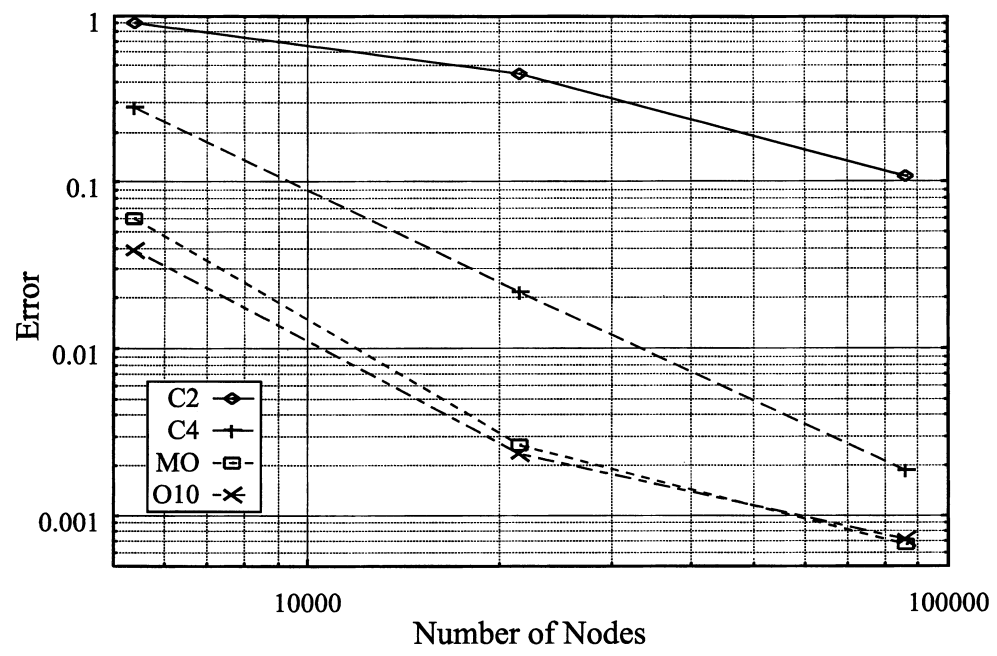

FIG. 13. $\mathrm{L}_{2}$ norm of the error in the electric field intensity as a function of the number of nodes in the grid for a cosine wave, with $\kappa=2 \pi$, incident on the perfectly conducting cylinder.

tained using the MO method is considerably more accurate than that obtained using the fourth-order method.

The optimized scheme does not show significant improvement over the maximumorder scheme in the above tests because of the relatively short distance of travel for the waves being considered. To test longer distances of travel (in terms of wavelength) a highly resolved grid with 345,600 nodes is used, and incident waves with much higher wavenumbers are modeled. With $\kappa=8 \pi$, the wave is resolved with 10 to $20 \mathrm{PPW}$ and travels 32 wavelengths during the simulation. For this case, the optimized method produces an error that is about $40 \%$ of that produced by the MO scheme. With 


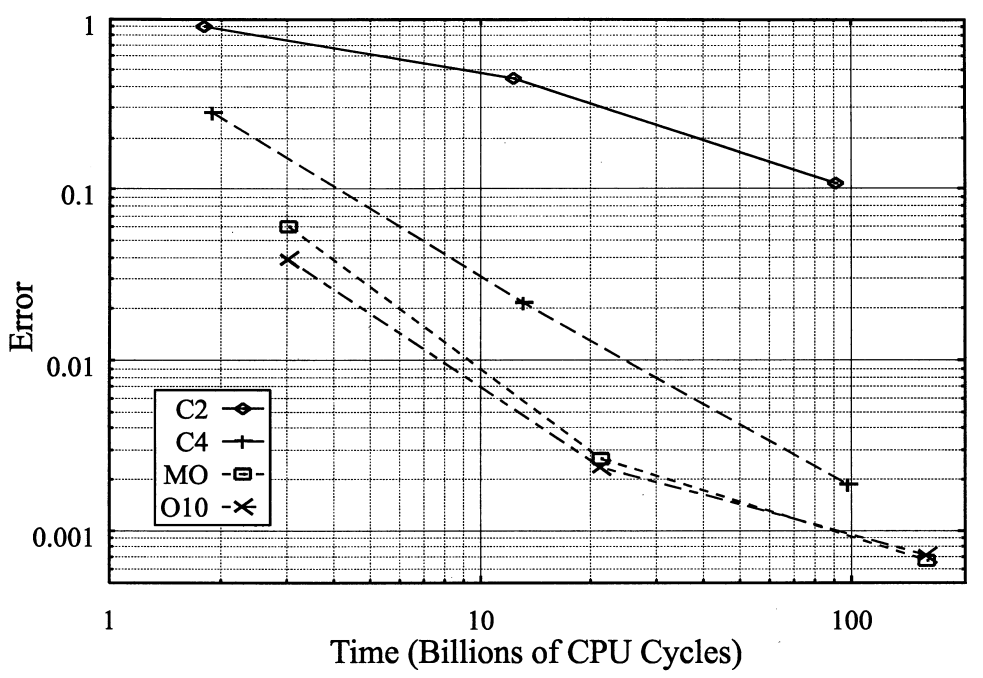

FIG. 14. $\mathrm{L}_{2}$ norm of the error in the electric field intensity as a function of the CPU time necessary to complete the simulation for a cosine wave, with $\kappa=2 \pi$, incident on the perfectly conducting cylinder.

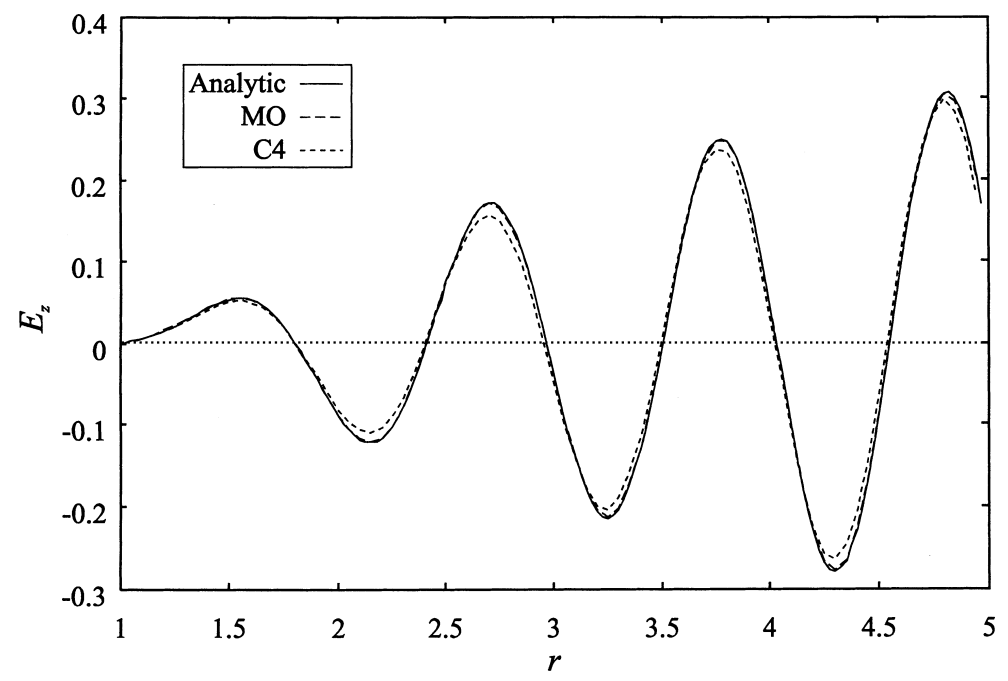

FIG. 15. Electric field intensity along a radial line behind the perfectly conducting cylinder for an incident cosine wave with $\kappa=2 \pi$.

$\kappa=10 \pi$, the resolution is 8 to $16 \mathrm{PPW}$, and the distance of travel is 40 wavelengths. For this wavenumber, the optimized method produces an error that is about $25 \%$ of that produced by the maximum-order scheme. This shows that the benefits of the optimized scheme increase as the number of wavelengths traveled between reflections increases.

6. Conclusions. Two high-accuracy finite-difference schemes have been used to solve the two-dimensional time-domain Maxwell equations to simulate electromagnetic wave propagation and scattering. The schemes have successfully modeled perfectly 
conducting and dielectric scatterers on Cartesian and curvilinear grids. The highaccuracy methods have been shown to be more efficient than classical second-order and fourth-order methods in terms of computing time and memory. The optimized scheme can produce some error reduction relative to the maximum-order scheme with no additional expense, especially when the number of wavelengths of travel is large. Overall, the results demonstrate that these methods are an efficient option for simulating the propagation and scattering of high-frequency electromagnetic waves.

Appendix. The following are the coefficients of the optimized scheme:

$$
\begin{aligned}
& a_{1}=0.759961, \quad a_{2}=-0.158122, \quad a_{3}=0.0187609 \\
& d_{0}=0.1, \quad d_{1}=-0.0763846, \quad d_{2}=0.0322896, \quad d_{3}=-0.005905, \\
& \alpha_{1}=0.168850, \quad \alpha_{2}=0.197348, \quad \alpha_{3}=0.250038, \quad \alpha_{4}=0.333306, \quad \alpha_{5}=0.5 .
\end{aligned}
$$

The following are the extrapolation and interpolation schemes used along interfaces:

$$
\begin{aligned}
u_{j} & =6 u_{j+1}-15 u_{j+2}+20 u_{j+3}-15 u_{j+4}+6 u_{j+5}-u_{j+6}, \\
u_{j+1 / 2} & =\left(3 u_{j-2}-25 u_{j-1}+150 u_{j}+150 u_{j+1}-25 u_{j+2}+3 u_{j+3}\right) / 256, \\
u_{j+1 / 2} & =\left(-7 u_{j-1}+105 u_{j}+210 u_{j+1}-70 u_{j+2}+21 u_{j+3}-3 u_{j+4}\right) / 256, \\
u_{j+1 / 2} & =\left(63 u_{j}+315 u_{j+1}-210 u_{j+2}+126 u_{j+1}-45 u_{j+4}+7 u_{j+5}\right) / 256, \\
u_{j} & =\frac{3}{4}\left(u_{j-1}+u_{j+1}\right)-\frac{3}{10}\left(u_{j-2}+u_{j+2}\right)+\frac{1}{20}\left(u_{j-3}+u_{j+3}\right) .
\end{aligned}
$$

The following can be used to calculate the absolute value of the flux Jacobians and the split flux Jacobians, for example, $|\hat{\mathbf{A}}|=|\hat{\mathbf{J}}(\xi)|$ and $\hat{\mathbf{B}}^{ \pm}=\hat{\mathbf{J}}^{ \pm}(\eta)$ :

$$
\begin{gathered}
|\hat{\mathbf{J}}(k)|=\left[\begin{array}{ccc}
c|\nabla k| & 0 & 0 \\
0 & c \frac{1}{|\nabla k|}\left(\frac{\partial k}{\partial y}\right)^{2} & c \frac{1}{|\nabla k|} \frac{\partial k}{\partial x} \frac{\partial k}{\partial y} \\
0 & -c \frac{1}{|\nabla k|} \frac{\partial k}{\partial x} \frac{\partial k}{\partial y} & c \frac{1}{|\nabla k|}\left(\frac{\partial k}{\partial x}\right)^{2}
\end{array}\right], \\
\hat{\mathbf{J}}^{ \pm}(k)=\frac{1}{2}\left[\begin{array}{ccc} 
\pm c|\nabla k| & \frac{1}{\mu} \frac{\partial k}{\partial y} & -\frac{1}{\mu} \frac{\partial k}{\partial x} \\
\frac{1}{\varepsilon} \frac{\partial k}{\partial y} & \pm c \frac{1}{|\nabla k|}\left(\frac{\partial k}{\partial y}\right)^{2} & \mp c \frac{1}{|\nabla k|} \frac{\partial k}{\partial x} \frac{\partial k}{\partial y} \\
-\frac{1}{\varepsilon} \frac{\partial k}{\partial x} & \mp c \frac{1}{|\nabla k|} \frac{\partial k}{\partial x} \frac{\partial k}{\partial y} & \pm c \frac{1}{|\nabla k|}\left(\frac{\partial k}{\partial x}\right)^{2}
\end{array}\right],
\end{gathered}
$$

where

$$
|\nabla k|=\sqrt{\left(\frac{\partial k}{\partial x}\right)^{2}+\left(\frac{\partial k}{\partial y}\right)^{2}} .
$$


The following are the local characteristic variables in the $\xi$ (use $k=\xi$ ) and $\eta$ (use $k=\eta$ ) directions:

$$
\begin{aligned}
& w_{+}=Z D_{z}+\left(\frac{\partial k}{\partial y} B_{x}-\frac{\partial k}{\partial x} B_{y}\right) /|\nabla k|, \\
& w_{-}=Z D_{z}-\left(\frac{\partial k}{\partial y} B_{x}-\frac{\partial k}{\partial x} B_{y}\right) /|\nabla k| .
\end{aligned}
$$

\section{REFERENCES}

[1] D. A. Anderson, J. C. Tannehill, And R. H. Pletcher, Computational Fluid Mechanics and Heat Transfer, Hemisphere, New York, 1984.

[2] A. Bayliss and E. Turkel, Radiation boundary conditions for wave-like equations, Comm. Pure Appl. Math., 33 (1980), pp. 707-725.

[3] J.-P. BERENGER, A perfectly matched layer for the absorption of electromagnetics wave, J. Comput. Phys., 114 (1994), pp. 185-200.

[4] M. H. Carpenter, D. Gottlieb, and S. Abarbanel, Time-stable boundary conditions for finite-difference schemes solving hyperbolic systems: methodology and application to highorder compact schemes, J. Comput. Phys., 111 (1994), pp. 220-236.

[5] B. Engquist And A. Majda, Absorbing boundary conditions for the numerical simulation of waves, Math. Comp., 31 (1977), pp. 629-651.

[6] Z. HARAS AND S. TA'ASAn, Finite difference schemes for long-time integration, J. Comput. Phys., 114 (1994), pp. 265-279.

[7] R. F. Harrington, Time-Harmonic Electromagnetic Fields, McGraw-Hill, New York, 1961.

[8] C. Hinsch, Numerical Computation of Internal and External Flows, Vol. 2: Computational Methods for Inviscid and Viscous Flows, Wiley, London, 1990.

[9] R. Hixon, S.-H. Shin, and R. R. Mankbadi, Evaluation of boundary conditions for computational aeroacoustics, American Institute of Aeronautics and Astronautics J., 33 (1995), pp. 2006-2012.

[10] O. HolBerg, Computational aspects of the choice of operator and sampling interval for numerical differentiation in large-scale simulation of wave phenomena, Geophysical Prospecting, 35 (1987), pp. 629-655.

[11] H. M. Jurgens, High-Accuracy Finite-Difference Schemes for Linear Wave Propagation, Ph.D. thesis, University of Toronto, ON, Canada, 1997.

[12] H. M. JuRGENS AND D. W. ZINGG, Implementation of a high-accuracy finite-difference scheme for linear wave phenomena, in Proceedings of the Third International Conference on Spectral and High Order Methods, Houston J. Math., Houston, TX, 1995, pp. 363-372.

[13] S. K. LelE, Compact finite difference schemes with spectral-like resolution, J. Comput. Phys., 103 (1992), pp. 16-42.

[14] Y. LIU, Fourier analysis of numerical algorithms for the Maxwell equations, J. Comput. Phys., 124 (1996), pp. 396-416.

[15] D. P. Lockard, K. S. Brentner, and H. L. AtKins, High-accuracy algorithms for computational aeroacoustics, American Institute of Aeronautics and Astronautics J., 33 (1995), pp. 246-251.

[16] A. H. Mohammadian, V. Shankar, and W. F. Hall, Computation of electromagnetic scattering and radiation using a time-domain finite-volume discretization procedure, Comput. Phys. Comm., 68 (1991), pp. 262-281.

[17] P. OLsson, Summation by parts, projections, and stability I, Math. Comp., 64 (1995), pp. $1035-$ 1065.

[18] P. G. Petropoulos, Phase error control for FD-TD methods of second and fourth order accuracy, IEEE Trans. Antennas and Propagation, 42 (1994), pp. 859-862.

[19] P. G. Petropoulos, L. Zhao, and A. C. Cangellaris, A reflectionless sponge layer absorbing boundary condition for the solution of Maxwell's equations with high-order staggered finite difference schemes, J. Comput. Phys., 139 (1998), pp. 184-208.

[20] J. S. Shang, A fractional-step method for the time domain Maxwell equations, J. Comput. Phys., 118 (1995), pp. 109-119.

[21] J. S. Shang And D. Gaitonde, On High Resolution Schemes for Time-Dependent Maxwell Equations, American Institute of Aeronautics and Astronautics paper 96-0832, 1996. 
[22] V. Shankar, A. H. Mohammadian, and W. F. Hall, A time-domain finite-volume treatment for the Maxwell equations, Electromagnetics, 10 (1990), pp. 127-145.

[23] A. Taflove, Computational Electrodynamics: The Finite-Difference Time-Domain Method, Artech House, Norwood, MA, 1995.

[24] C. K. W. TAm and J. C. WebB, Dispersion-relation-preserving finite difference schemes for computational acoustics, J. Comput. Phys., 107 (1993), pp. 262-281.

[25] E. Turkel And A. Yefet, Fourth order method for Maxwell equations on a staggered mesh, IEEE Antennas and Propagation Society International Symposium 1997 Digest, 4 (1997), pp. 2156-2159.

[26] R. Vichnevetsky and J. B. Bowles, Fourier Analysis of Numerical Approximations of Hyperbolic Equations, SIAM, Philadelphia, 1982.

[27] B. Yang, D. Gottlieb, and J. S. Hesthaven, Spectral simulations of electromagnetic wave scattering, J. Comput. Phys., 134 (1997), pp. 216-230.

[28] K. S. YEE, Numerical solution of initial boundary value problems involving Maxwell's equations in isotropic media, IEEE Trans. Antennas and Propagation, 14 (1966), pp. 302-307.

[29] J. L. Young, D. Gaitonde, And J. S. Shang, Toward the construction of a fourth-order difference scheme for transient EM wave simulation: Staggered grid approach, IEEE Trans. Antennas and Propagation, 45 (1997), pp. 1573-1580.

[30] D. W. ZINGG, Comparison of high-accuracy finite-difference methods for linear wave propagation, SIAM J. Sci. Comput., 22 (2000), pp. 476-502.

[31] D. W. ZingG And T. T. Chisholm, Runge-Kutta methods for linear ordinary differential equations, Appl. Numer. Math., 31 (1999), pp. 227-238.

[32] D. W. ZingG, P. D. Giansante, And H. M. Jurgens, Experiments with High-Accuracy FiniteDifference Schemes for the Time-Domain Maxwell Equations, American Institute of Aeronautics and Astronautics paper 94-0232, 1994.

[33] D. W. ZINGG AND H. Lomax, On the eigensystems associated with numerical boundary schemes for hyperbolic equations, in Numerical Methods for Fluid Dynamics, M. J. Baines and K. W. Morton, eds., Clarendon Press, Oxford, UK, 1993, pp. 473-481.

[34] D. W. ZingG, H. Lomax, And H. Jurgens, High-accuracy finite-difference schemes for linear wave propagation, SIAM J. Sci. Comput., 17 (1996), pp. 328-346.

[35] D. W. ZingG, H. Lomax, And H. Jurgens, An Optimized Finite-Difference Scheme for Wave Propagation Problems, American Institute of Aeronautics and Astronautics paper 93-0459, 1993. 